\title{
EE2 IMPACTS INNATE IMMUNE PATHWAYS IN ZEBRAFISH
}

\author{
Siba R. Das, ${ }^{12}$, James C. Woodson ${ }^{1}$, John D. Hansen ${ }^{1,2 S}$ \\ ${ }^{1}$ U.S. Geological Survey, Western Fisheries Research Center, Seattle, WA. \\ ${ }^{2 *}$ Pacific Northwest Diabetes Research Institute, Seattle, WA. \\ ${ }^{3}$ Department of Global Health, University of Washington, Seattle, WA.
}

Worldwide, there are serious concerns regarding the impacts of endocrine disrupting chemicals in aquatic environments due to their potential to alter physiological processes for vertebrate health. One of the most frequently detected endocrine disrupting compounds, $17 \alpha$-ethinylestradiol (EE2), is found in a variety of aquatic ecosystems at ng $\mathrm{L}^{-1}$ concentrations. EE2 can severely alter physiological processes including development and reproduction but very little research has addressed the impact of EE2 on the immune system of fish. Recently, we assessed the impact of EE2 on the immune mRNA transcriptome in adult zebrafish by sub-chronically exposing fish to EE2 followed by a 24-hour immune stimulation period with peptidoglycan and poly(I:C) to mimic microbial infection. This microarray study identified multiple innate immune pathways that were down-regulated upon exposure to EE2 implying that EE2 likely suppresses innate immunity. Additionally, the analysis suggested the involvement of miRNAs for regulating some of these pathways. To address this observation, we used RNAseq to profile miRNAs from the EE2/PAMP experiment described above. Individual miRNA libraries $(n=36)$ were sequenced at a depth of more than $10 \mathrm{M}$ reads per individual fish. Raw reads were filtered, trimmed, aligned to the miRBase 21 database and counts were then normalized to assess differentially expressed miRNAs. Putative gene targets were identified from the miRBase target database and correlated with the previous microarray results. Overall, our results suggest that both mRNAs and miRNAs participate in the dysregulation of innate immunity through the impacts of EE2.

Key Words: zebrafish, innate, PAMP, miRNA, EE2

${ }^{\S}$ Corresponding author: Tel.: 001.206.526.6588; Fax: 001.206.526.6654

*Current address

E-mail address: John D. Hansen, jhansen@usgs.gov 
